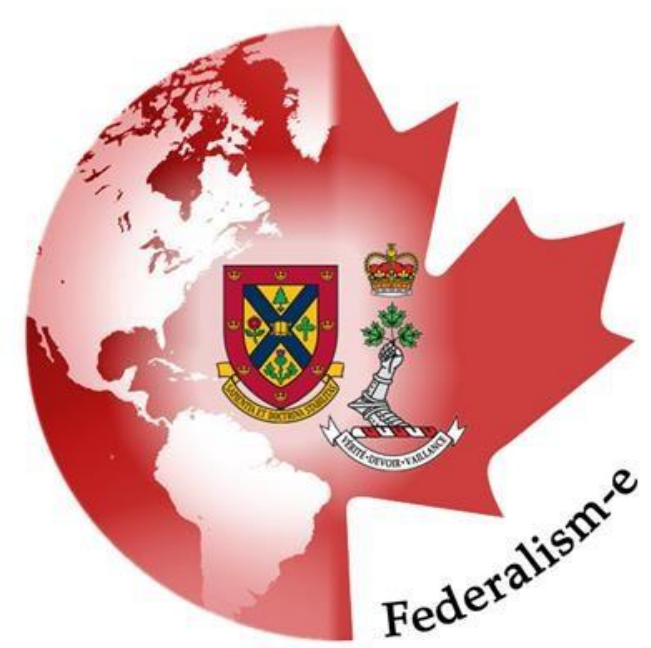

\title{
Diviser pour mieux régner : faut-il décentraliser les pouvoirs?
}

\author{
Alek Robaczewski, Collège militaire royal du Canada
}

C'est en 1867 que John A. Macdonald, désirant former un gouvernement canadien indépendant, conclut qu'une union fédérale serait la seule option viable. (Pelletier R. et Tremblay M., 2009, p.18) C'est ainsi que fut écrite la Loi constitutionnelle de 1867, qui octroyait aux provinces canadiennes un gouvernement provincial avec ses propres sphères d'influence, sous la supervision d'un gouvernement fédéral, lui-même titulaire d'une panoplie de pouvoirs. Ce dernier fut constitué à la britannique : à sa tête, la Reine ou le Roi (et son représentant, le Gouverneur Général), soutenu par le pouvoir exécutif et le pouvoir législatif, le premier étant subordonné au second. La législation devait être établie par la chambre des Communes, constituée de députés élus, ainsi que par la chambre des Lords, aujourd'hui des sénateurs nommés au Sénat, ayant pour objectif de réviser et peaufiner le travail de la chambre des Communes. Le pouvoir exécutif, quant à lui, devait être tenu par le Premier Ministre et son cabinet. Finalement, il est à noter que la triade de la gouvernance fut complétée par le pouvoir judiciaire, tenu par la cour suprême du Canada et ayant pour tâche la médiation et l'interprétation des lois. (Gouvernement du Canada, « Loi constitutionnelle de $1867 »)$ 
Bien qu'elle ne fasse pas preuve de beaucoup d'innovation comparativement à la métropole britannique, cette constitution permettait d'instaurer des gouvernements pouvant facilement accomplir les objectifs des pères fondateurs du Canada : la maintenance de la stabilité et de l'ordre. Cela devait se faire par l'imputabilité de l'exécutif face au législatif. Toutefois, aujourd'hui, l'équilibre des forces tend à s'inverser, la Chaire de recherche sur la démocratie et les institutions parlementaires de l'Université Laval en soulevant un symptôme: "[...] le Sénat canadien a toujours eu de la difficulté à jouer pleinement son rôle de seconde chambre fédérale ». (Université Laval)

Bien que ce soit un problème considérable, étant donné le rôle du sénat dans la machine gouvernementale, je crois cela n'est qu'un indice d'un déficit démocratique encore plus grand: la centralisation et la concentration des pouvoirs vers une seule instance. C'est, à mon avis, là que se trouve la plus grande faille du système de gouvernement $d u$ Canada et que toute réforme future devrait aspirer à compenser pour celle-ci, dans le but d'éventuellement ramener l'équilibre original entre le pouvoir exécutif et législatif.

Pour supporter cette thèse, seront d'abord étudiées les implications et conséquences de la concentration du pouvoir, pour poursuivre par une observation des avantages qu'apporterait une modification du mode électoral. Finalement, les arguments pour une réforme du sénat seront discutés dans un but de combattre ce que Pierre Mackay décrit comme "[un sénat] dont la légitimité démocratique est proche du coefficient zéro". (Mackay P., 2006-2007, p.63.)

\section{Un pouvoir concentré}

Tout d'abord, en théorie, tout corps exécutif canadien est sous l'autorité de la Reine d'Angleterre et de son représentant au Canada, le gouverneur général. Bien que les deux rôles existent encore aujourd'hui et que, sur papier, ils dirigent le Canada, en pratique, ils agissent plutôt à titre honorifique. Il est en effet reconnu, comme le dit J.P. Lewis, qu'au Canada de nos jours, le Premier Ministre est tout puissant et qu'il a, en pratique et sous certaines conditions, un contrôle immense sur la politique canadienne.(Lewis J.P., 2013, p.799 à 819)

En effet, les députés élus à la chambre des Communes appartiennent pour la très grande majorité à des partis politiques, eux-mêmes dirigés par un chef. Lorsque ce parti est 
celui ayant le plus de députés à la chambre basse, ses membres gagnent l'accès au gouvernement et leur dirigeant devient Premier Ministre. Un gouvernement élu majoritaire, c'est-à-dire ayant obtenu moins de 154 sièges à la chambre des Communes, implique que plus de la moitié des personnes qui votent sur les lois à être légiférées adhèrent à un ensemble d'idéologies et d'objectifs semblables, et ce, en plus d'être soumis à une discipline de parti qui tend à être serrée. Ce dernier aspect, d’importance capitale, est largement une conséquence $d u$ fait que le chef d'un parti est celui qui décide qui sera ministre, incitant les élus mineurs à appuyer ses intérêts s'ils désirent grimper les échelons du pouvoir et assurant leur rectitude dans l'adhésion aux lignes partisanes. (Kam C.J. 2009, p.29)

De fait, la tête de parti et ses proches ont peu de problèmes à faire passer leurs idées à la chambre basse et il ne reste plus qu'à obtenir l'approbation du Sénat. Bien que celui-ci soit censé être constitué " d'éminences grises " neutres et impartiales, les sièges vacants sont alloués à des personnes nominées par le gouverneur général, après recommandation du Premier Ministre. Évidemment, ce dernier y installe des sénateurs sympathiques à sa cause, et une polarisation semblable à celle de la chambre des Communes vient colorer le Sénat selon les partis politiques majeurs. Les projets y étant soumis ont d'ores et déjà peu de chance d'être balayés. En somme, le plus haut responsable du gouvernement, tenant du pouvoir exécutif, acquiert à travers sa mainmise sur le parti le contrôle du pouvoir législatif, tant à la chambre haute qu'à la chambre basse.

\section{Vers une meilleure représentation}

Plus largement, le phénomène de concentration du pouvoir n'est pas seulement observable au niveau individuel chez les chefs partis. En effet, d'un point de vue macroscopique, celui-ci se remarque chez les partis eux-mêmes, à travers la surreprésentation des partis majeurs et la sous-représentation des partis moins importants. Ceci est attribuable au système d'élection fédéral, où tous les sièges de la chambre des Communes sont alloués aux élus de chaque circonscription. La problématique qui en découle est plus fortement observable chez les petits acteurs politiques, qui ont tendance à recueillir des voix un peu partout mais à avoir très peu (ou aucun) élus, bien souvent en raison de campagnes publicitaires plus faibles. Inversement, ce sont les partis 
majeurs ayant d'énormes budgets qui amassent ces sièges et il en résulte des déséquilibres entre le nombre de députés et le pourcentage de popularité d'un parti.(Cross W., 2011, p.142) Afin de rééquilibrer le jeu, plusieurs entités, notamment la Commission du droit du Canada, proposent de modifier le mode de scrutin et de migrer vers un système mixte.(Des Rosiers N. et al., 2009) Le modèle écossais peut être utilisé à titre d'exemple, ayant un peu plus de la moitié de ses députés élus par comtés, le reste étant choisis selon la proportion de votes obtenus par un parti.(Gray I., Gray J., 2004, p.21) Le tableau suivant, tiré de la Revue Parlementaire canadienne, illustre d'ailleurs ce modèle s'il était appliqué au Canada:

\section{TABLEAU 1:}

Les élections fédérales de 2004 et les résultats découlant d'un système mixte

\begin{tabular}{|c|c|c|c|c|c|}
\hline & \multicolumn{3}{|c|}{$\begin{array}{l}\text { Résultats des élections fédérales de } \\
2004\end{array}$} & \multicolumn{2}{|c|}{$\begin{array}{l}\text { Résultats du modèle } \\
\text { écossais }\end{array}$} \\
\hline & $\begin{array}{l}\% \text { du vote } \\
\text { populaire }\end{array}$ & $\begin{array}{l}\mathrm{N}^{\mathrm{bre}} \mathrm{d} \\
\text { e sièges }\end{array}$ & $\begin{array}{l}\% \mathrm{de} \\
\text { sièges }\end{array}$ & $\begin{array}{l}\mathrm{N}^{\text {bre }} \mathrm{de} \\
\text { sièges }\end{array}$ & $\begin{array}{l}\% \mathrm{de} \\
\text { sièges }\end{array}$ \\
\hline Libéral & $36,7 \%$ & 135 & $\begin{array}{l}43,8 \\
\%\end{array}$ & 120 & $\begin{array}{l}38,6 \\
\%\end{array}$ \\
\hline $\begin{array}{l}\text { Conser } \\
\text { vateur }\end{array}$ & $29,6 \%$ & 99 & $\begin{array}{l}32,1 \\
\%\end{array}$ & 96 & $\begin{array}{l}30,9 \\
\%\end{array}$ \\
\hline NPD & $15,7 \%$ & 19 & $6,2 \%$ & 47 & $\begin{array}{l}15,1 \\
\%\end{array}$ \\
\hline $\begin{array}{r}\text { Bloc } \\
\text { Québécois }\end{array}$ & $12,4 \%$ & 54 & $\begin{array}{l}17,5 \\
\%\end{array}$ & 38 & $\begin{array}{l}12,2 \\
\%\end{array}$ \\
\hline Vert & $4,3 \%$ & 0 & $0,0 \%$ & 9 & $2,9 \%$ \\
\hline Autre & $1,3 \%$ & 1 & $0,3 \%$ & 1 & $0,3 \%$ \\
\hline & & 308 & & 311 & \\
\hline
\end{tabular}

Source: GRAY Ian, GRAY James. "La représentation proportionnelle : le modèle écossais appliqué aux élections canadiennes de 2004 », Revue Parlementaire canadienne, volume 27, n³, 2004, p.23, tableau 2. 
Parmi les conclusions qui peuvent être tirées de ce tableau, c'est que les partis plus importants auraient généralement tendance à perdre des sièges au profit des partis moins bien représentés, le cas du NPD et du parti Vert étant les plus notables (il est intéressant de noter que le parti régional qu'est le Bloc Québécois perdrait aussi de l'influence, car ne pouvant représenter qu'une petite partie de la population canadienne et étant particulièrement dépendant de ses circonscriptions). De fait, les partis Conservateur et Libéral auront une plus grande difficulté à acquérir une majorité sur les autres, tout en ayant à faire face à une opposition plus forte et diverse. Ultimement, affaiblir les grands partis implique une moins grande concentration de pouvoir vers ceux-ci et donc, leur chef. En somme, l'implantation d'un système mixte pourrait contribuer à réduire la concentration des pouvoirs tout en offrant un mode d'élection plus représentatif de la popularité réelle des partis et donc plus démocratique.

\section{Un Sénat plus neutre et impartial}

Dans un autre ordre d'idées, le Sénat est régulièrement dans la mire des critiques du gouvernement et l'argument de son illégitimité règne. (Smith, D. E., 2003, p.58) En effet, le fait que ses membres soient nommés fait en sorte qu'il y a une forte corrélation entre le gouvernement au pouvoir et l'allégeance politique de ceux-ci. Par ailleurs, tel qu'expliqué par Pelletier et Tremblay dans leur ouvrage, la chambre haute n'a jamais vraiment accompli l'un des aspects pour lequel il a été instauré, soit la représentation dans l'arène centrale des acteurs fédérés. (Pelletier R. et Tremblay M., 2009, p.348) Un remaniement du Sénat permettrait de faire d'une pierre deux coups et d'apporter une solution à ces deux problèmes. Une démarche pourrait être de modifier son fonctionnement afin que ceux y siégeant soient nommés par les gouvernements provinciaux plutôt que par le parti fédéral au pouvoir. De plus, leur accorder un mandat beaucoup plus court, en allouant une quantité de sièges par province selon la proportion de sa démographie par rapport à celle du Canada, y assurerait une plus grande fluidité des idées.

La logique d'où découle cet argument est que si un gouvernement provincial a été élu, c'est que la population de sa province y a accordé sa confiance et croit qu'il prendra de bonnes décisions de gouvernance, notamment concernant les nominations. De plus, cela retirerait le pouvoir de sélection des mains du Premier Ministre, sans pour autant 
provoquer une dilution de l'expertise qui pourrait résulter d'une élection, où la popularité d'un candidat est plus importante que sa compétence. Les nommés n'auraient donc plus de comptes à rendre au gouvernement et seraient plus impartiaux face aux projets proposés par celui-ci, tout en garantissant à chaque entité fédérée une représentation au niveau central. Cela donnerait une chance au Sénat d'accomplir efficacement (et pour la première fois) son second mandat. Il faudrait toutefois considérer généraliser l'obligation pour chacun de se détacher de tout lobby ou parti politique, autant national que provincial, avec lesquels il pourrait y avoir des affiliations, à l'image de ce qu'a fait le Parti Libéral de Justin Trudeau. (Les Sénateurs libéraux siégeront comme indépendants) D’autre part, mandater par province les sénateurs permettrait de rapprocher le Sénat du publique. En effet, ses membres auraient à être remplacés bien plus fréquemment par les différents gouvernements provinciaux, qui sont en théorie plus près de leurs électeurs que le pallier fédéral, impliquant donc plus directement le citoyen dans le processus de sélection qu'auparavant. Finalement, ces mesures permettraient de garantir un Sénat se renouvelant constamment.

\section{Conclusion}

En somme, je crois que le plus grand déficit démocratique au Canada se trouve dans la concentration des pouvoirs vers des partis élitistes et puissants, qui eux-mêmes canalisent ceux-ci vers un groupe très sélect et restreint de gens permanents et influents à la tête de ces organisations. Cela a plusieurs conséquences, notamment de donner des élections moins représentatives de l'opinion réelle de la population, d'élire des députés représentant un parti au détriment de la circonscription qui l'a élevé ainsi que de fournir une grande quantité de pouvoirs discrétionnaires et de sélection au le Premier Ministre (nomination des chefs des sociétés d'état, des juges, des sénateurs, etc.). Instaurer un mode d'élection mixte, incluant des sièges accordés de manière proportionnelle, réduirait l'influence desdits grands partis. Dans un autre ordre d'idées, faire en sorte que les sénateurs soient nommés par les gouvernements provinciaux favoriserait la diversité d'opinion en amenuisant les allégeances envers les partis fédéraux, et ce, en permettant au Sénat de mieux accomplir son mandat. Ultimement, ces ajustements contribueraient à 
faire du gouvernement canadien un leadership plus représentatif en rétablissant l'équilibre entre le pouvoir législatif et exécutif, formant une institution plus démocratique.

\section{$\underline{\text { Bibliographie }}$}

CROSS, William. Political Parties, Vancouver, UBC Press, 2011, 206 pages.

DES ROSIERS N., et al. Un vote qui compte : la réforme électorale au Canada, Commission du droit du Canada, 2009, 256 pages.

GOUVERNEMENT DU CANADA. «Loi constitutionnelle de 1867 », [en ligne], 14 mars 2014, Site web de la législation du Canada, http://laws-lois.justice.gc.ca/fra/Const/, (13 avril 2014)

GOUVERNEMENT DU CANADA. « Loi constitutionnelle de 1982 », [en ligne], 14 mars 2014, http://lawslois.justice.gc.ca/ fra/const/page-16.html\#h-53, (13 avril 2014).

GRAY, Ian et James GRAY. "La représentation proportionnelle : le modèle écossais appliqué aux élections canadiennes de $2004 »$, Revue Parlementaire canadienne, volume 27, n³, 2004, p.21 à 24.

KAM, Christopher J. Party Discipline and Parliamentary Politics. Cambridge, Cambridge University Press, 2009, 278 pages.

LEWIS, J.P. «Elite Attitudes on the Centralization of Power in Canadian Political Executives: A Survey of Former Canadian Provincial and Federal Cabinet Ministers, 2000-2010», Canadian Journal of Political Science, volume 46, 2013, p.799 à 819.

MACKAY, Pierre. "Le fédéralisme et le partage des compétences législatives », Notes de cours JUR2515, Droit constitutionel, UQAM, Chapitre 3, 2006-2007.

PARLEMENT DU CANADA. « Le parlement canadien», [en ligne], 2011, Site web du parlement du Canada, http://www.parl.gc.ca/About/Parliament/GuideToHoC/index-f.htm, (13 avril 2014).

PELLETIER, Réjean et Manon TREMBLAY. Le parlementarisme canadien, Québec, Les presses de l'Université Laval, 2009, 581 pages.

SHIVELY, W. Phillips et Jules-Pascal VENNE. Pouvoir et décision, Montréal, Chenelière McGraw-Hill, 2008, 239 pages.

SMITH, D.E. The Canadian Senate in Bicameral Perspective, Toronto, University of Toronto Press, 2003, 272 pages.

UNIVERSITÉ LAVAL. « 8e congrès québécois de droit constitutionnel - La réforme du Sénat », [en ligne], s.d., Site de la Chaire de recherche sur la démocratie et les institutions parlementaires de l'Université Lava., http://www.democratie.chaire.ulaval.ca/index.php?pid=1084\&n=702, (17 avril 2014). 